\title{
(Does mathematics provide a model for the philosophy of computer science?) Idealism and Lisp and Perl.
}

\author{
Camille Akmut
}

We argue that in computer science, programming languages is one area where the question of epistemological idealism may arise : we propose that Lisp can be considered representative of what we call a "language waiting to be discovered", as we put, while Perl conforms more closely to the model of a language created; additional examples from the extended domain of software are included. 


\section{Introduction}

Does mathematics provide a model for the philosophy of computer science?

A portion of today's computer scientists like to flatter themselves by conceiving of their existences as next-of-kin mathematicians; a sentiment sometimes accompanied by a horror of the humanities, while mathematicians have their own ideas about such conceptions...

Yet even the most mathematics-heavy areas such as cryptography continuously show them wrong, to say nothing of more obvious regions like human-computer interaction.

Public-private key cryptography, to give one example, is as much a major theoretical achievement as a well-known and tolerated practical catastrophe.

The best of encryption is worthless if users fail to use it - does this sound like mathematics? A matter at least equally for sociologists, quasi-mathematicians be displeased. .

During the first lesson of a famous recorded lecture, Harold Abelson can be seen striking the word science out of "computer science";

Russell observed in his 'Introduction' that "matters ... were included in philosophy so long as no satisfactory science of them existed."

This paper represents the attempt to gather ideas somewhere in-between, "on the frontier of knowledge", uncertain and doubly necessary.

An expose of mathematical idealism is followed by applications of these ideas to the field of programming languages within computer science.

Attempts to find further illustrations in other software occur.

The computer scientist, unlike the mathematician of the early twentieth c., need not apologize : the consequences of their theories and abstractions are but everywhere to be seen. 


\section{Mathematical platonism}

We begin by briefly recalling the main philosophies of mathematics that are platonism, its variants and opposites;

A classic definition of platonism in mathematics has been provided by Bernays as follows : "to view [mathematical] objects as detached/independent from the thinking subject" (trans.) (Bernays 1935 : 53).

Russell's Introduction to mathematical philosophy begins with the series of natural numbers, with the author noting that their emergence through abstraction must have taken a long time and is the product or sign of an advanced civilization starting with 1 , and even more so $0 \ldots$

Coming after them, Haskell Curry described platonism as the view that "ascribes an independent existence" to conceptions (precisely "to all the infinistic conceptions of classical mathematics") (Curry 1951:6).

Among idealistic views of mathematics, he distinguishes further intuitionism, which as its name suggest puts an emphasis on intuition within mathematics (Curry $1951: 6$ ).

Curry also noted elsewhere, "Probably platonism is the view adopted, more or less subconsciously, by most mathematicians who do not concern themselves explicitly with foundational questions. It is also the position of the pioneers in mathematical logic, Frege ... and Russell, and it is defended today by some of the ablest logicians." (Curry 1977 : 9)

In a modern review of these debates, Bernays is described as distinguishing between "strong" and "weak" variants of platonism :

the weakest of them consists in admitting the existence of the series of natural numbers (already opposed by intuitionists like Kronecker) while absolute platonism postulates a world of ideal objects holding all mathematical objects and relationships (deprecated by the Russel-Zermelo paradoxes). ${ }^{1}$

Bernays writes "modest" (Bernays 1935 : 54).

Bouveresse argues that the main opposition between the various schools lies not as much in the ontological problems (of independent existences) as what means we posses to identify mathematical objects and which mathematical objects we can reach with them (Bouveresse 1999 : 369).

Next, the programming languages Lisp* and Perl are considered and examined as illustrations of these ideas.

[We discuss mainly Common Lisp and Scheme. $\left.{ }^{2}\right]$

Barski 2011.

\footnotetext{
${ }^{1}$ See Bouveresse 1999 : 357.

${ }^{2}$ For an introduction, refer to
} 


\section{Lisp : a language waiting to be discovered?}

\subsection{Design}

Quoted from the 1.5 documentation :

"In the LISP language, all data are in the form of symbolic expressions usually referred to as S-expressions. (...) In the LISP programming system, the bulk of available memory is used for storing S-expressions in the form of list structures." (McCarthy 1962)

The minimalism of Lisp's design lends it all the characteristics of a language that could be described as waiting to be discovered.

Its manual is barely 50 pages long.

"Lisp only has one way of organizing bits of code: It uses parentheses to organize the code into lists." - Barski adds that "all basic Lisp code uses this simple list-like syntax" (Barski 2011 : 33)

He goes on to explain how "Common Lisp uses two modes when it reads your code: a code mode and a data mode." (ibid. : 35) (the default being code)

That lists play a central role - LISP standing for list processor ${ }^{3}$ - is apparent from the perspective of the user but it is also mirrored on the side of the program :

Per the PL textbook 'Programming language pragmatics', which is a more formal presentation :

"A program in Lisp is itself a list" ("and can be manipulated with the same mechanisms used to manipulate data") of programs and data".

Elsewhere Scott writes in more detail of this :

"a program in Scheme takes the form of a list. In technical terms, we say that Lisp and Scheme are homoiconic: self-representing. A parenthesized string of symbols (in which parentheses are balanced) is called an S-expression regardless of whether we think of it as a program or as a list. In fact, a program is a list, and can be constructed, de-constructed, and otherwise manipulated with all the usual list functions." (ibid.)

The minimalism of this design should not be taken to mean simplicity, if simplicity implies some limitations.

So-called "macros" play an important role in this regard, leading the author of Let Over Lambda to express the extreme, but not solitary view that "all other languages are just skins on top of lisp."

\footnotetext{
${ }^{3}$ Coll. GNU Emacs Lisp Reference Manual. "Lisp history".

${ }^{4}$ (First ed.) Ch. 11, "Nonimperative programming models".
} 
"Macros are the single greatest advantage that lisp has as a programming language and the single greatest advantage of any programming language. With them you can do things that you simply cannot do in other languages. Because macros can be used to transform lisp into other programming languages and back, programmers who gain experience with them discover that all other languages are just skins on top of lisp."

(See also Greenspun's tenth rule.)

Scott notes that "modern languages and compilers have, for the most part, abandoned macros as an anachronism", but that in part. Scheme and Common Lisp have taken the alternative, safe approach of hygienic macros.

\subsection{Culture}

In popular culture, Lisp is most often associated with

-- wizards, magicians....,

-- religion / mysticism,

The menorah included in official versions of CLISP, displayed at every REPL start, or "The ancient and beautiful Chinese ying-yang" (per Guy Steele in "the little LISPer').

-- aliens,

Most prominently perhaps in the logo, cover, illustrations of the book Land of Lisp.

-- (solitary) genius, general otherworldliness,

A recent iteration of this idea is found in e.g. 'The Perfect Insider', whose ending segment or ED features a Lisp implementation of Conway's game of life.

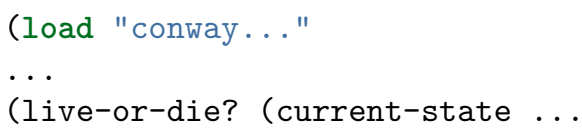

A more classic example is offered by 'Serial experiments Lain'; episode 7 has fragments of a program (being either the same, or what looks to be a similar project) while the teenage girl protagonist's bedroom is increasingly filled with, at least partly mysterious, electronics.

(defun life (source) ...

(life (make-world) ... 
The principal function of these associations is to suggest an object discovered, rather than created or invented.

A visitor from another planet becomes known. A genius sees what others don't. Religious truths are revealed, through various means (visions, rituals, introspection/meditation, interpretations of texts considered holy... ${ }^{5}$ ). Finally, an apprentice witch learns the rules of nature, and to use them, she has not made them or the universe.

There are, laid out, some of the elements that participate to the fairly consistent and repeating imagery found in the cultural representations of Lisp.

These various evocations are not random : they coincide in some way with the scientific and technological characteristics of this small language made of little more than nested parentheses.

Additionally, the books The little LISPer and Schemer take on the traits of some Socratic question-answer-and-are-you-really-sure dialog.

Figures 1 and 2 complement this iconography.

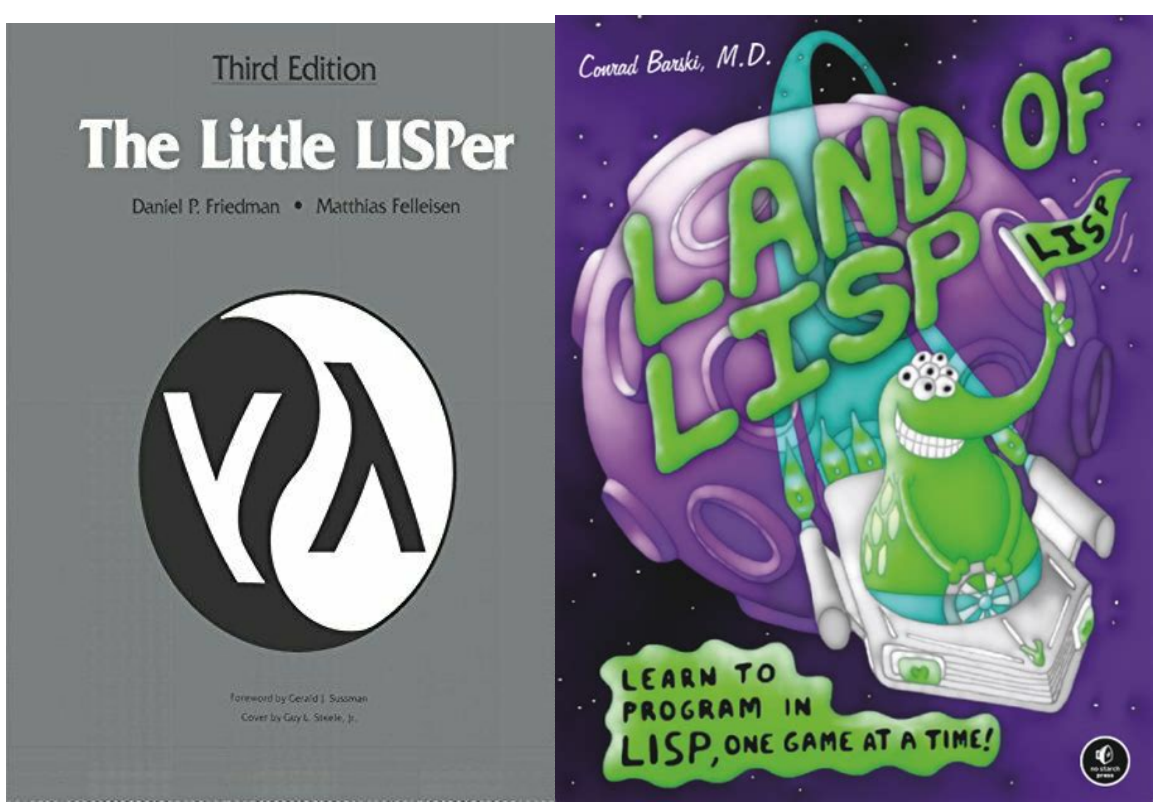

Figure 1: Covers of two popular Lisp books.

\footnotetext{
${ }^{5}$ Religions have their own independent notions of history, truth, logic... e.g. in the Gospels, the temptation of Christ; John the Baptist's sect etc.
} 


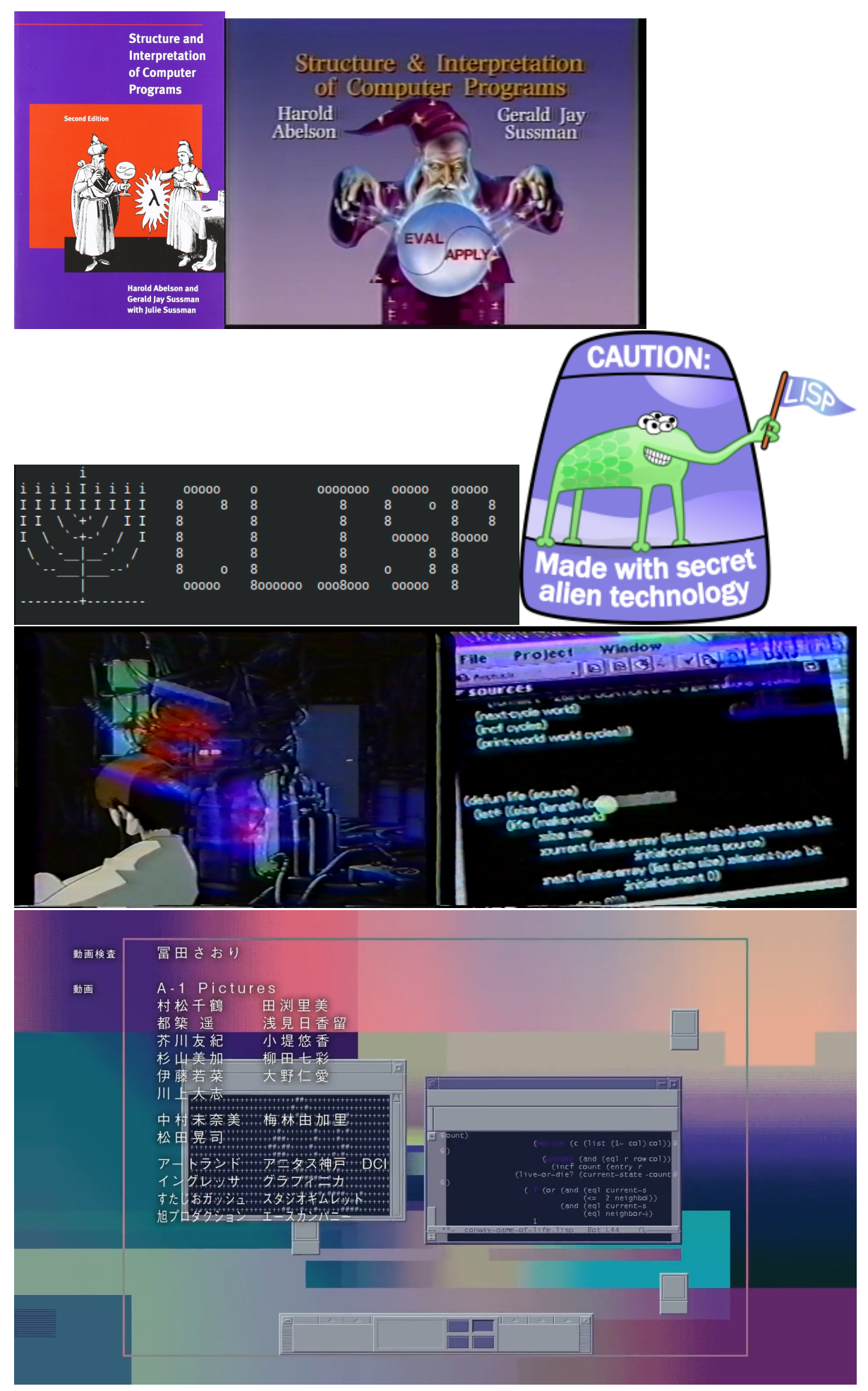

Figure 2: Representations (cont.) - SICP; CLISP; "Alien Lisp"; Lain; P. I.. 


\section{Perl}

Perl is a creation deeply tied to a community, which is to say an inventor and users;

sometimes this distinction is not absolutely clear, as users become creators or co-creators themselves (a fact enabled by free software).

Its inventor Larry Wall co-wrote the reference manual, Programming Perl, whose preface reads :

Perl's magic comes from many sources: the utility of its feature set, the inventiveness of the Perl community, and the exuberance of the open source movement in general. But much of this magic is simply hybrid vigor; Perl has a mixed heritage and has always viewed diversity as a strength rather than a weakness. Perl is a "give me your tired, your poor" language. If you feel like a huddled mass longing to be free, Perl is for you. Perl reaches out across cultures. Much of the explosive growth of Perl has been fueled by the hankerings of former Unix systems programmers who wanted to take along with them as much of the "old country" as they could. For them, Perl is the portable distillation of Unix culture, an oasis in the desert of "can't get there from here".

In other words, here is a language described as a melting pot of cultures.

To be complete, it is presented in many other ways as well including as a language with a "split personality"... (at the same time simple and rich) (See Wall et al. 2000)

Perl may well be the world's most personal language.

Its willed, or at least encouraged idiosyncrasies, lead to code that is almost as unique as the individual behind it.

e.g.

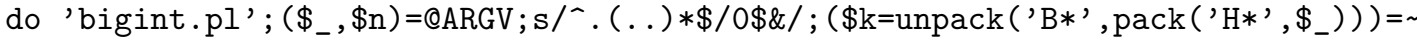
$\mathrm{s} / \sim 0 * / / ; \$ \mathrm{x}=0 ; \$ \mathrm{z}=\$ \mathrm{n}=\sim \mathrm{s} / . / \$ \mathrm{x}=\operatorname{Lbadd}(\& \mathrm{bmul}(\$ \mathrm{x}, 16)$, hex $\$ \&) / \operatorname{ge} ;$ while (read (STDIN, $\$$, $\$ \mathrm{w}$ $=((2 * \$ \mathrm{~d}-1+\$ \mathrm{z}) \& \sim 1) / 2))\left\{\$ \mathrm{r}=1 ; \$=\operatorname{substr}\left(\${ }_{-} \cdot " \backslash 0 " \mathrm{x} \$ \mathrm{w}, \$ \mathrm{c}=0, \$ \mathrm{w}\right) ; \mathrm{s} / . \mid \mathrm{n} / \$ \mathrm{c}=\& \mathrm{badd}(\& \mathrm{bmul}\right.$

$(\$ \mathrm{c}, 256)$, ord $\$ \&) / \mathrm{ge} ; \$=\$ \mathrm{k} ; \mathrm{s} / . / \$ \mathrm{r}=\& \operatorname{bmod}(\& \mathrm{bmul}(\$ \mathrm{r}, \$ \mathrm{r}), \$ \mathrm{x}), \$ \& ? \$ \mathrm{r}=\& \operatorname{bmod}(\& \mathrm{bmul}(\$ \mathrm{r}, \$ \mathrm{c}$

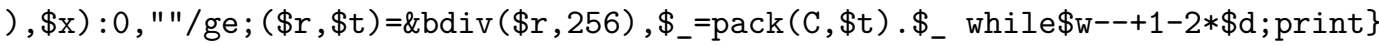

Figure 3: RSA in Perl, http://www.cypherspace.org/rsa/pureperl.html

For more such "one-liners", a tradition popular enough in the Perl world that a monograph on the subject was written, see for instance Krumins 2013.

Finally, our analysis is extended to the wider domain of existing software. 


\section{Software (brief remarks on Emacs)}

In software, an extension of programming languages, similar patterns of idealism and anti-idealism are found.

The computer software Emacs is an interesting example of this, for holding components of both philosophies :

On one hand the idea of - what could be called - a 'total system', one enabling all tasks, does not contradict platonism, i.e. such a notion is not very personal, in other words it is not tied to any specific individual. ${ }^{6}$

On the other, its default configuration is one of the most striking products of individualism in software, testimony of a unique personality at work.

To start using Emacs feels like a slow descent into a very specific and particular type of mind.

The average user - like any person of average laziness - may find the use of one modifier self-evident; in this software the combination of multiple modifier sequences (e.g. M-x + M-y) is implied.

However this software is highly customizable and should make overriding this initial configuration possible; remaining a most opinionated system without modifications.

[None of the above remarks are meant to say anything about the quality of the software, whether one way or the other.]

\footnotetext{
${ }^{6}$ Although it is probably representative of a certain personality from the psychological viewpoint. .
} 
Ref.

* Bernays, Paul. 1935. "Sur le platonisme dans les mathematiques" L'enseignement mathematique 34.

* Curry, Haskell. 1951. Outlines of a formalist philosophy of mathematics. North-Holland pub.

"In the opening days of September, 1939, just as the Second World War was breaking out, an International Congress for the Unity of Science was held at Cambridge, Massachusetts. [It was] suggested that I write out, in a more or less systematic form, the philosophical opinions which I had formed (...) This book deals with the philosophy of mathematics, not from the point of view of a philosopher, but from that of a mathematician who has had occasion to reflect on the nature of his science."

* Curry, Haskell. 1977. Foundations of mathematical logic. Dover.

(A discussion of platonism is found in part. in chap. 1)

* Russell, Bertrand. 1920. Introduction to mathematical philosophy. 2nd ed..

"It is the purpose of this book to explain mathematical philosophy simply and untechnically (...) A full treatment will be found in Principia Mathematica"

* Bouveresse, Jacques. 1999. "Sur le sens du mot "platonisme" dans l'expression "platonisme mathematique"" Revue de theologie et de philosophie 131(4) : 353370 .

* McCarthy, John. 1962. 'LISP 1.5 Programmer's Manual'.

* Scott, Michael. Programming language pragmatics. 4 editions so far.

* Grant et al.. 2020. Principles of programming languages.

* Collective. 'SICP'. MIT Press.

(In part. ch. 5, implementation; ch. 4, a "metacircular evaluator" i.e. a Lisp interpreter written in Lisp. ${ }^{7}$ )

* Wall, Larry et al.. 2000. Programming Perl. O'Reilly. 3rd ed..

(The second edition features about the same preface, if not the same.)

* Barski, Conrad. 2011. Land of Lisp. No Starch Press.

* Hoyte, Doug. 2008. Let Over Lambda.

* Krumins, Peteris. 2013. Perl one-liners. No Starch Press.

* Coll. The little LISPer. (3rd ed.)

\footnotetext{
${ }^{7}$ For a reference on metacircular interpreters, or so-called "evaluators", see e.g. Grant et al. ("Metacircular interpreters give you some idea of how a language works, but suffer from ... non-foundational problems").
} 